\section{Modeling in the Classroom: Making Relationships and Systems Visible}

\author{
Kristy J. Wilson, ${ }^{* *}$ Tammy M. Long, ’ Jennifer L. Momsen, $\$$ and Elena Bray Speth" \\ 'Biology Department, College of Arts and Sciences, Marian University, Indianapolis, IN 46222; \\ "Department of Plant Biology, Michigan State University, East Lansing, MI 48823; 'Department \\ of Biological Sciences, North Dakota State University, Fargo, ND 58108; "Biology Department, \\ Saint Louis University, St. Louis, MO 63103
}

\begin{abstract}
As an instructional tool, models can transform the student experience from the static to the dynamic, the flat to the 3D, and the siloed to the integrated. Few practical resources exist to help instructors transition toward model-based classroom practices. The Modeling in the Classroom evidence-based teaching guide provides instructors with a tool kit for incorporating models and modeling into their classrooms (https://lse.ascb .org/evidence-based-teaching-guides/modeling-in-the-classroom). The guide discusses the underpinnings of modeling as a core scientific practice, one that can enable student development of systems thinking skills and understanding of biological concepts. The guide describes a variety of model types, including phylogenetic trees, simulations, animations, diagrams, conceptual models, concept maps, and tactile models supported by summaries of and links to articles and resources. In this paper, we will introduce key findings describing why and how to use models in the classroom. We also describe open research questions needed to address classroom implementation, instructional design, and development of students' knowledge and skills. It is our hope that the guide will provide a suitable combination of research-based findings and practical suggestions that instructors will be supported and encouraged to thoughtfully incorporate modeling to support learning goals.
\end{abstract}

\section{INTRODUCTION}

It would be hard to imagine a practice more integral to science than modeling. Indeed, some have even defined science as, "a process of constructing predictive conceptual models" (Gilbert, 1991). Scientists model in order to visualize and organize their thinking, to distill complex ideas into simpler representations that can be shared with and evaluated by peers or communicated to others, to test hypotheses, and identify gaps in our understanding. Not surprisingly, science learners also benefit from using and creating models to develop and connect their knowledge as they learn within a discipline. Model-based instruction (MBI) that engages students in using, building, revising, and evaluating models can promote multiple dimensions of science learning, including both development of content understanding and skills (Schwarz et al., 2009; Louca and Zacharia, 2012). Despite the foundational role of models in science and the robust evidence base underpinning $\mathrm{MBI}$, models and modeling are not well represented in curricula or instruction designed to train future biologists, particularly at the undergraduate level.

Recognizing that few practical resources exist to help instructors transition toward model-based classroom practices, we developed the evidence-based teaching guide (EBTG) Modeling in the Classroom (https://lse.ascb.org/evidence-based-teaching -guides/modeling-in-the-classroom). The EBTG provides instructors a tool kit for incorporating models and modeling into their classrooms and highlights core ideas, important considerations, and key examples related to MBI (Figure 1). In this guide, we present modeling as a scientific practice and show how modeling promotes
CBE Life Sci Educ March 1, 2020 19:fe1 DOI:10.1187/cbe.19-11-0255

*Address correspondence to: Kristy J. Wilson (kjwilsonamarian.edu).

○ 2020 K. J. Wilson et al. CBE-Life Sciences Education (๑) 2020 The American Society for Cell Biology. This article is distributed by The American Society for Cell Biology under license from the author(s). It is available to the public under an Attribution-Noncommercial-Share Alike 3.0 Unported Creative Commons License (http://creativecommons.org/licenses/ by-nc-sa/3.0)

"ASCB®" and "The American Society for Cell Biology $\circledR^{\prime \prime}$ are registered trademarks of The American Society for Cell Biology. 


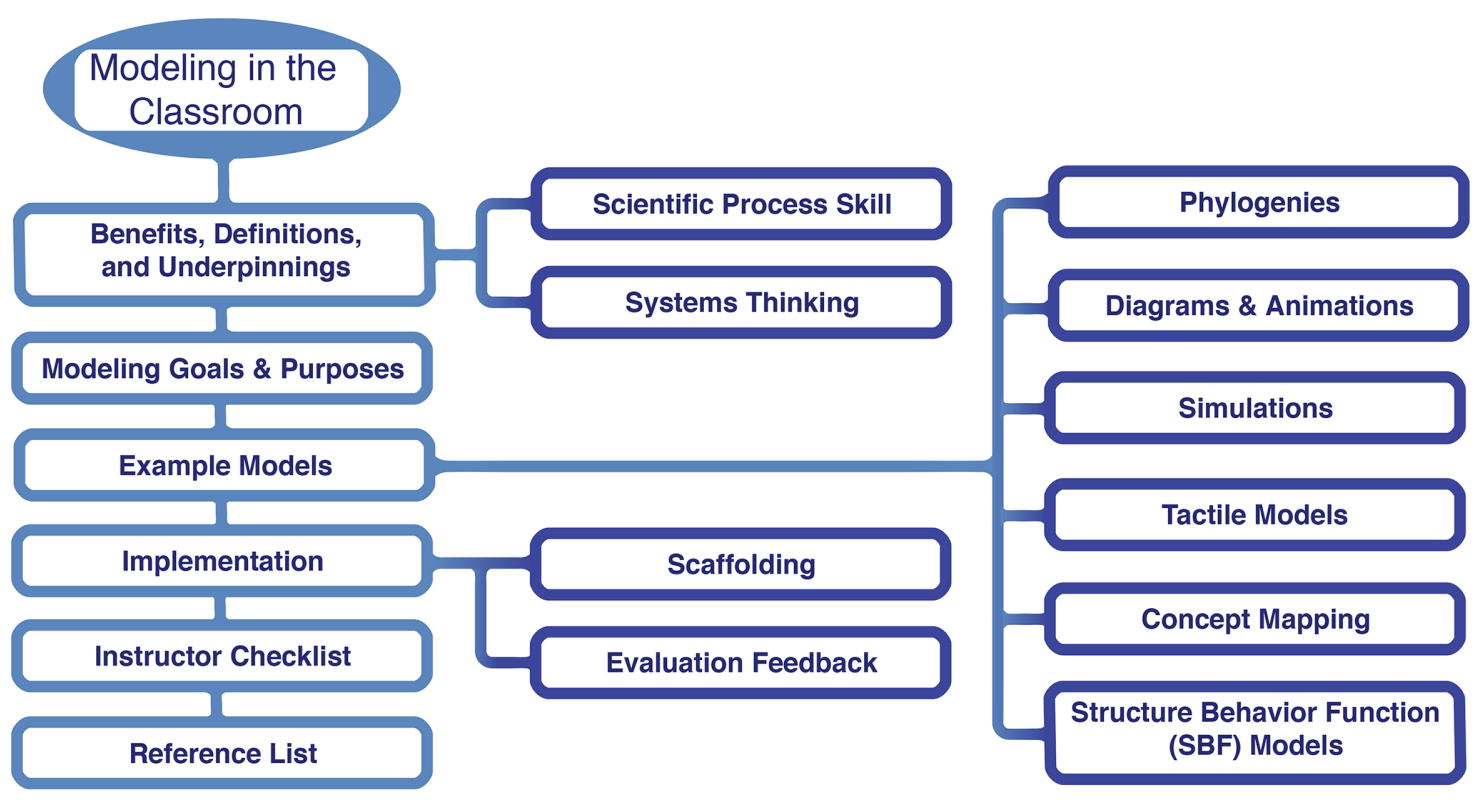

FIGURE 1. Screenshot representing the landing page of the Modeling in the Classroom guide. This provides an overview of choice points.

scientific and systems thinking, and how modeling can serve diverse learning goals across a range of biological subjects and subdisciplines. We also provide recommendations for scaffolding modeling instruction and delivering feedback that promotes and strengthens students' development of model-based skills. In addition, the guide includes a selection of model types that are commonly used in biology instruction, including phylogenetic trees, simulations, animations, diagrams, conceptual models, concept maps, and tactile models (Figure 2). Each core idea and model type is supported by a brief summary of key research findings and actionable advice intended to help instructors make evidence-based decisions about classroom instruction. Links to select articles direct readers to original research, and an Instructor Checklist details considerations and recommendations to help instructors incorporate modeling into their classroom practice. It is our hope that the guide will provide a suitable combination of research-based findings and practical suggestions so that instructors will find both encouragement and support when considering, adding, or refining modeling in their instruction. In this essay, we 1) introduce the main ideas represented in the modeling teaching guide, and 2) discuss needs and opportunities for research on modeling instruction and students' modeling skills, especially at the undergraduate level.

\section{WHY USE MODELS IN A CLASSROOM?}

Our experiences as instructors of introductory biology underpin a desire to create learning opportunities that allow students to see and understand the complexities of life. After all, biology is more than memorizing disconnected facts. Organizing one's biological knowledge, however, can be overwhelming. Modeling can help scientists and learners question, organize, and visualize their understanding. The process of creating and evaluating models may help learners develop and reinforce connections between seemingly disparate ideas, resulting in deep and long-lasting learning. Thus, modeling can support learning goals related to biology content, making content more versatile and transferable for students. Like scientists, students can also use models to make and test predictions, explain phenomena, or communicate research results.

Models and MBI can also support the development of systems thinking skills. Although we currently lack a framework describing the systems thinking skills life science students should develop, it is clear that modeling can support students as they learn to describe and reason about biological systems. In particular, modeling can support students as they learn to identify and describe a system of interest-the boundaries of the system, the elements comprising the system, and how those elements interact.

Finally, modeling can be a component of formative and summative assessment. Students can interpret given models in multiple-choice or short-answer questions to address the central role models play in the discipline. Students can create novel models to demonstrate content understanding as well as modeling skills. By having students create models for formative or summative evaluation, we allow students to express their thoughts through different modes, thus making the classroom more inclusive. Additionally, student-generated models have great utility in revealing student difficulties, particularly as they relate to mechanistic explanations. These models allow instructors to provide rapid, individualized, and specific feedback to promote both better modeling and conceptual understanding (Evaluation Feedback section in the Modeling in the Classroom Guide).

\section{HOW SHOULD INSTRUCTORS IMPLEMENT MODEL-BASED INSTRUCTION?}

Articulating clear and specific learning goals should lead to making instructional decisions that are consistent with the intended learning outcomes. This would include choices in model selection that would support specific learning objectives; modeling should not be added just for the sake of modeling but 

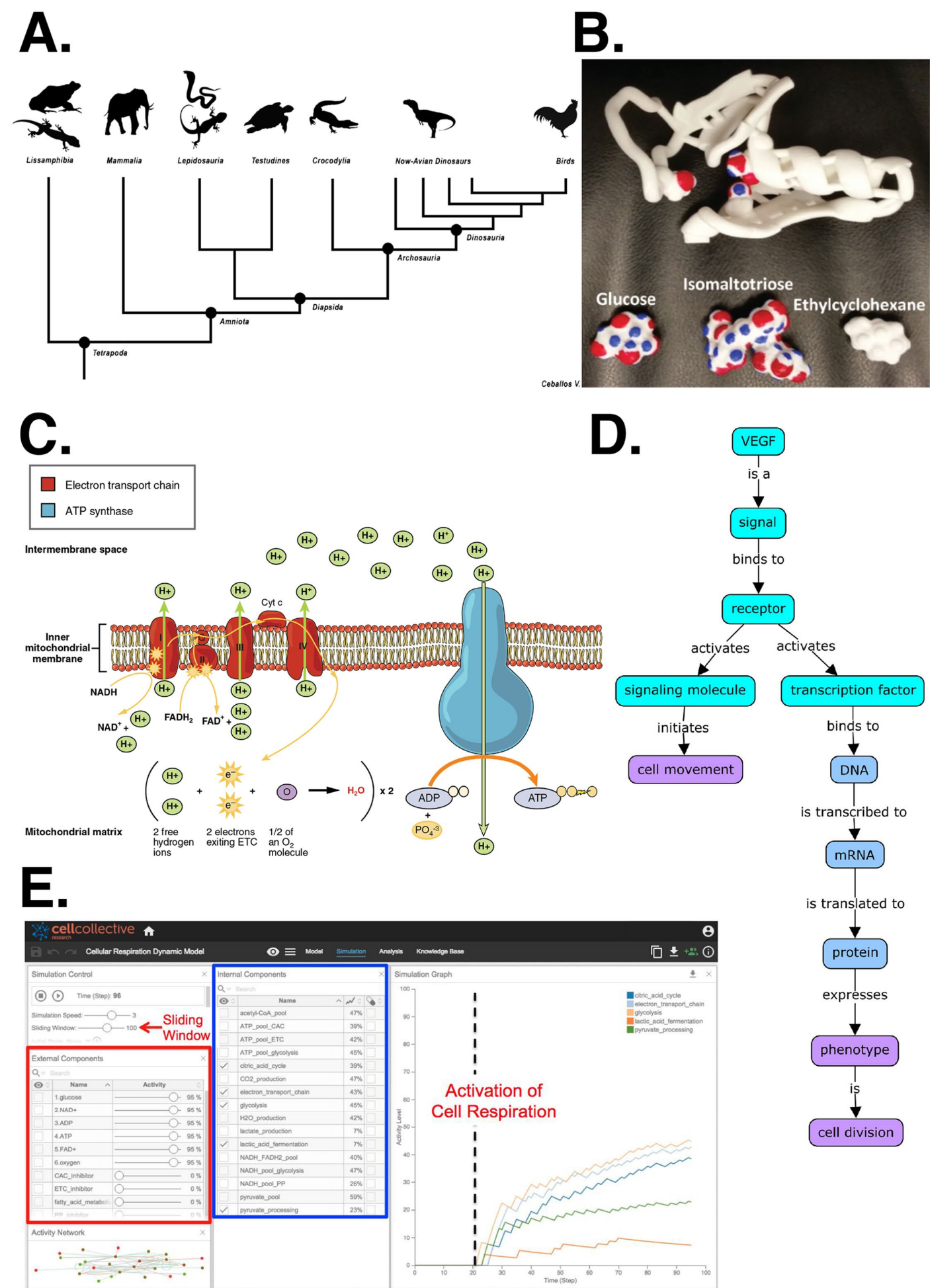

FIGURE 2. Examples of model types described in the guide. (A) Example phylogeny in recommended branched format (Novick and Catley, 2018). Figure shared under Creative Commons Attribution-ShareAlike 4.0 International License (Ceballos, 2018). (B) Fused-filament tactile model to allow visualization of noncovalent interactions to rationalize enzyme substrate complementarity. Reproduced from BabiloniaRosa et al. (2018) with permission from the Royal Society of Chemistry. (C) Process diagram of electron transport chain and oxidative phosphorylation that supports understanding membrane role, appearance of proteins, and how reactions occur. Figure presented under Creative Commons 3.0 license (OpenStax College, 2013). (D) Example student-created conceptual model using structure-behaviorfunction formatting with signaling (teal), gene expression (blue), and phenotype (purple). (E) Simulation window from Cell Collective showing model components in colored boxes, the simulation control sliding window, and the simulation graph of cellular respiration (Bergan-Roller et al., 2017). Figure shared under Creative Commons Attribution-ShareAlike 4.0 International License. 
deliberately tied to learning goals. For example, phylogenies can be used to demonstrate relationships between organisms, and tactile models can show structure-function relationships in macromolecules. Ideally, depending on the learning goals, classroom activities may involve students using and interpreting given models, or creating, evaluating, and revising their own models.

Skills required for interpreting, using, and building models are not intuitive, but can be developed with practice and instruction. Instructors cannot assume that models transparently convey meaning for students (Schönborn and Anderson, 2009,2010 ). Students need guidance on how to read and interpret each new model they encounter, even if the only difference is in formatting (Wright et al., 2018; Scaffolding section in the Modeling in the Classroom Guide). Thus, when implementing model-based instruction, planning sufficient time and activities for instruction, practice, and feedback is essential. Deliberate practice and scaffolding of modeling activities allow students to acquire and use skills and build on these skills through increasingly complex tasks (Hobbs et al., 2013). Modeling is especially suited for small-group work, as it allows students to cooperatively communicate what and why certain components are needed in a model and how those components work together in the context of the modeled system (Scaffolding section in the Modeling in the Classroom guide).

The modeling skills students practice in the course of instruction should be assessed in ways that are consistent with the learning objectives, which may require departure from conventional, closed-response tests as students interpret or construct models. Instructors' feedback should similarly be aligned with one's learning goals for modeling and should address both content knowledge and modeling skills. For concrete steps to incorporate modeling into a course, please see recommendations throughout the Modeling in the Classroom guide and in its associated Instructor Checklist.

\section{OPEN RESEARCH QUESTIONS}

While many principles of model-based teaching and learning have been identified, research on model-based instruction in the college biology classroom is a growing field that still holds much room for progress and discovery. Important areas that require more research include best practices of instructional design and classroom implementation, as well as model-based learning of concepts and acquisition of science process skills.

\section{Classroom Implementation and Instructional Design}

Multiple kinds of models (Example Models section of the Modeling in the Classroom guide) are relevant in biology, including qualitative conceptual models of processes and pathways, quantitative models and simulations, three-dimensional models of molecular or anatomical structures, phylogenies representing hypotheses about evolutionary relationships, and more. Further research is necessary to build upon what is currently known from research on learning progressions (Schwarz et al., 2009) to identify which types of models and modeling skills should be included at different stages of undergraduate biology education. Research should also help educators define learning goals, design instruction and assessment, and optimize approaches for providing feedback to learners. Student-generated models, used as a type of assessment, can provide information about students' conceptual understanding of a topic, but also about achievement of modeling skills and abilities-a core Vision and Change competency of undergraduate biology education.

Providing meaningful and actionable feedback to students about their work is a critical component of instruction and is still generally an area in need of research. As a result, there is limited evidence about how best to evaluate and provide feedback to students on their models or on the process of modeling itself. When students construct models, it is particularly challenging for instructors to articulate feedback about both the form (model organization and structural features) and the substance (biological content) of these artifacts. Form and content are intricately connected components of models, which likely affect one another. In light of these considerations, multiple research questions are still to be explored, including:

- What kinds of models should undergraduate biology students be able to create, interpret, and use at different stages in the curriculum?

- What should the goals of model-based instruction be? Should modeling be used to help students develop deep conceptual understanding of biology, to teach specific modeling skills, or perhaps a combination of both?

- How should instructors introduce and scaffold classroom activities to promote students' fluency with models and modeling? Should instruction about different types of models (e.g., simulations, phylogenetic trees, conceptual models) be scaffolded in different ways?

- When (e.g., for what kinds of models and what core concepts of biology) is it appropriate for students to build models, as opposed to using provided models?

- How can modeling be effectively and efficiently integrated into assessment approaches?

- How can we structure feedback on modeling such that it is meaningful and actionable for students?

\section{Development of Knowledge and Skills}

Creating and using models are core scientific practices. Scientists use models for a variety of purposes depending on what they are communicating, to whom, and for what purpose. While it is clear that models and modeling have important cognitive benefits for learners, we are only beginning to explore how model-based learning can foster students' understanding of the nature of science, support deeper conceptual understanding of content, and promote systems thinking. For example, learners struggle to build connections within and across biological systems and to reason about system dynamics, causality, and emergence. Modeling may promote development of these reasoning skills, which are part of the broader frame of thinking referred to as "systems thinking." While the field of biology education research acknowledges the importance of systems thinking skills, there is no consensus on what comprises systems thinking or how to assess systems thinking skills in the undergraduate classroom. In light of these considerations, multiple research questions are still to be explored, including:

- Under what conditions could model-based learning foster students' development of science process skills (making hypotheses and predictions, articulating causal explanations, etc.)? 
- How can we teach students to evaluate models and to identify model limitations and constraints?

- How can the integration of modeling in a biology curriculum support not only content understanding, but also development of systems thinking skills? How do we measure competence in systems thinking?

\section{CONCLUSION}

Models can illustrate hypotheses, generate predictions, propose mechanisms, represent research findings, or describe the current understanding of structure and function of complex systems (Modeling Goals \& Purposes section of the Modeling in the Classroom guide). As an instructional tool, models can transform the student experience from the static into the dynamic, the flat to the $3 \mathrm{D}$, and siloed to integrated. Models have the potential to change students' view of the biology classroom from one in which they memorize lists of facts and take tests to one in which they learn to represent and reason about biological systems that are dynamic, multidimensional, integrated, and complex.

The contents of this guide reflect our collective interests and experiences using models and modeling in our classrooms for more than a decade. Initially, modeling provided a way for us to step away from multiple choice and toward assessments that more closely reflect authentic scientific practice. We soon came to value the ways in which models capture and condense students' thinking and to appreciate modeling as more than a skill to develop in our students. Modeling has become an approach for how we deliver instruction and a method for introducing and connecting new ideas. We have found that modeling activities make us mindful to demonstrate links between ideas and avoid the expert blind spot. We are more careful to communicate our thought processes, making them more accessible to students learning the material for the first time. We make concerted efforts to specify relationships in material covered, avoid making leaps between steps, and create opportunities for students to do the same. Through modeling, we can see how students approach the material of our courses and then give feedback to address deficiencies.

Modeling is now an indispensable and nonnegotiable component of our instruction. While the research on modeling in undergraduate biology continues to grow, we believe there is strong support for including modeling as part of undergraduate biology instruction. In our experiences, we have seen students use modeling techniques from our classrooms to study for other, non-model based classes and develop research plans. We propose that modeling should be incorporated throughout a curriculum to help students understand the profound role that models play in our discipline and to encourage them to use models for purposes well beyond our classrooms.

Ultimately, the goal of this guide is to help you, our fellow instructors, incorporate modeling into your classrooms in ways that serve your specific needs. Whether it is helping your students externalize their thinking, practice quantitative reasoning, predict and explain system behaviors, or evaluate their understanding of foundational biological processes, modeling is a flexible and valuable tool for accommodating myriad instructional goals.

\section{REFERENCES}

Babilonia-Rosa, M. A., Kouns, H. K., \& Oliver-Hoyo, M. T. (2018). Using 3D printed physical models to monitor knowledge integration in biochemistry. Chemistry Education Research and Practice, 19(4), 1199-1215.

Bergan-Roller, H. E., Galt, N. J., Dauer, J. T., \& Helikar, T. (2017). Discovering cellular respiration with computational modeling and simulations. Course Source, 4, 1-8.

Ceballos, V. G. (2018, September 28) Tetrapoda cladogram. Retrieved October 27, 2019, from https://commons.wikimedia.org/wiki/File:TEtrapoda _Cladogram.jpg

Gilbert, S. W. (1991). Model building and a definition of science. Journal of Research in Science Teaching, 28(1), 73-79.

Hobbs, F. C., Johnson, D. J., \& Kearns, K. D. (2013). A deliberate practice approach to teaching phylogenetic analysis. CBE-Life Sciences Education, 12(4), 676-686.

Louca, L. T., \& Zacharia, Z. C. (2012). Modeling-based learning in science education: Cognitive, metacognitive, social, material and epistemological contributions. Educational Review, 64(4), 471-492.

Novick, L. A., \& Catley, K. M. (2018). Teaching tree thinking in an upper level organismal biology course: Testing the effectiveness of a multifaceted curriculum. Journal of Biological Education, 52, 66-78.

OpenStax College (2013, June 19) 2508 electron transport chain. Retrieved June 3, 2019, from https://cnx.org/contents/FPtK1zmha6.27:nWir -Uwua3/Carbohydrate-Metabolism

Schönborn, K. J., \& Anderson, T. R. (2009). A model of factors determining students' ability to interpret external representations in biochemistry. International Journal of Science Education, 31(2), 193-232.

Schönborn, K. J., \& Anderson, T. R. (2010). Bridging the educational research-teaching practice gap: Foundations for assessing and developing biochemistry students' visual literacy. Biochemistry and Molecular Biology Education, 38(5), 347-354.

Schwarz, C. V., Reiser, B. J., Davis, E. A., Kenyon, L., Achér, A., Fortus, D., ... \& Krajcik, J. (2009). Developing a learning progression for scientific modeling: Making scientific modeling accessible and meaningful for learners. Journal of Research in Science Teaching, 46(6), 632-654.

Wright, L. K., Cardenas, J. J., Laing, P., \& Newman, D. L. (2018). Arrows in biology: Lack of clarity and consistency points to confusion for learners. CBE-Life Sciences Education, 17(1), ar6. 